\title{
Outline for an Operational Semantics of PromelA
}

\author{
V. Natarajan and Gerard J. Holzmann
}

\begin{abstract}
Promela is a high-level specification language for modeling interactions in distributed systems, and for expressing logical correctness requirements about such interactions. The model checker SPIN accepts specifications written in this language, and it can produce automated proofs for each type of property. SPIN either proves that a property is valid in the given system, or it generates a counter-example that shows that it is not. This paper contains the outline for an operationalsemantics definition of PROMELA.
\end{abstract}

\section{Introduction}

Promela is a language for reasoning about concurrent systems. The language has developed over the last fifteen years from a simple modeling language into a more complete specification language. The language, supported by the model checker SPIN, is used in a growing number of design projects, both large and small.

As new constructs are introduced into the language (e.g., [Hol95]), their interplay with existing constructs can have subtle consequences. Some combinations of constructs can reasonably be interpreted in more then one way. So far, only the implementation of the model checker SPIN can provide definitive answers about semantics issues. A formal semantics definition can provide an implementation independent way to resolve possible conflicts of interpretation.

This paper gives an outline for a semantics basis of the language. To simplify the exposition, it imposes a number of restrictions. It will not discuss, for instance, array variables or structures, nor will it discuss assert statements, never claims, or correctness properties expressed by progress and accept labels. To simplify the definitions somewhat, we will also restrict run statements to one single parameter, and channel declarations to a single message field.

1991 Mathematics Subject Classification. Primary 68Q65, 68Q22, 68M15; Secondary $68 \mathrm{~N} 20$.

Key words and phrases. Verification, Semantics, Promela, SPIN.

Work done during the first author's stay at Bell Laboratories in the summer of 1995.

(C) 1997 American Mathematical Society 
The remainder of this paper is organized as follows. Section 2 introduces notational conventions, and some semantic preliminaries. Section 3 formalizes the notion of executability of Promela statements. Section 4 presents how global states are transformed by the execution of statements. The notion of the execution of a program is captured in Section 5. Section 6 gives a small example of the conversion of a program into a labeled transition system. Section 7 summarizes the report.

\section{Preliminaries}

A PROMELA program consists of zero or more global variable declarations, and one or more process type declarations (proctypes). The verifier SPIN translates each process type declaration into a labeled transition system, in which the labels are primitive statements [Hol94]. We define below what a primitive statement is. For the time being it will suffice to note that a primitive statement is a state transformer: when it is executable (to be defined) it modifies the current state (to be defined) in a precise way (to be defined). Note that compound statements (such as if .. $f$, do .. od, unless (introduced in [Hol95]), and goto are not primitive statements, and neither are else statements or declarations.

Converting compound statements, or generally process type declarations, into labeled transition systems is a fairly straightforward procedure that is not detailed here. (It will be detailed in the full version.) Labeled transition systems are formally defined in Section 2.4. An example of the translation from a proctype declaration into a labeled transition system is given in Section 6. The conversion is performed by SPIN when it parses its input. The result can be seen after a verifier is generated and compiled, with: pan -d. (A graphical representation can also be seen with the FSM View option in XSPIN.)

2.1. Notational Conventions. If $S$ is a nonempty set then $S^{i}$ will denote the set of sequences of length $i$ whose elements belong to $S$. If $x$ is a sequence, then $x . i$ denotes the $i$ th element of $x$. We will often assign symbolic names to specific positions in a sequence; if sym is the symbolic name for the $i$ th position of the sequence $x$ then $x . s y m$ will denote $x . i$. If $x$ is any mathematical structure then $x[y / s y m]$ will stand for the same structure in which the substructure denoted by $s y m$ is replaced by $y$. If $f$ is a function then $f[s y m=y]$ will denote the function which takes the value $y$ at sym and for all other arguments has the same value as $f$. If $x$ is a sequence and $y$ is some element then $x \circ y$ is the sequence obtained by appending $y$ to the end of $x$. If $x$ is a sequence then $c d r(x)$ is the sequence obtained from $x$ by deleting its first element.

2.2. Syntactic Entities. We define the following syntactic entities: Vars, Consts, Exprs, Asgns, SendExprs, RecExprs and RunExprs.

Vars is the set of all variable names.

Consts is the set of all constants.

The term var refers to an element of Vars, const refers an element of Consts and convar refers to an element of either Vars or Consts.

Exprs is the set of expressions. 
The term expr refers to an element of Exprs. The elements of Exprs are defined by the context-free grammar:

$$
\begin{aligned}
& \text { bop }::=+|-| *|>|<|E Q| \mathrm{NE}|\mathrm{AND}| \mathrm{OR} \\
& \text { uop }::=-\mid ! \\
& \text { exp }::=\text { convar } \mid \text { (expr) | expr bop expr } \mid \text { uop exp } \mid \\
& \text { LEN var | timeout } \mid \text { var?[convar] }
\end{aligned}
$$

The next four sets define different types of statements, or state transformers. Asgns is the set of assignment statements, with elements of the form 'var $=e x p r$ '.

SendExprs is the set of send statements, with elements of the form 'var! expr'.

RecExprs is the set of receive statements, with elements of the form 'var? convar'.

RunExprs is the set of run statements, with elements of the form run Name expr'.

DEFINITION 2.1. A primitive statement is an element of one of the sets: Exprs, Asgns, SendExprs, RecExprs, or RunExprs.

Note that we have restricted run statements to a single parameter (in addition to the proctype name) and similarly we have restricted send and receive operations (and thereby channel declarations) to a single message field. These restrictions are meant to improve the readability of the definitions that follow.

2.3. Data Types and Value Domains. BTypes is the set of basic types, which we restrict here to $\{$ bit, byte, short, int $\}$. Types is the set of types given by BTypes $\cup\{$ chan $\}$.

To ensure that only finite state programs are defined, PROMELA sets finite upper bounds on certain parameters. We introduce symbolic names for these upper bounds. MaxChanCap is the maximum number of messages a channel can hold (all channels have finite capacity). MaxNumProc is maximum number of processes that can be active at a time, and MaxNumChan is the maximum number of channels that can be accessible at a time. For notational convience, we also define the following sets.

MaxChanCapSet $=\{i \mid 0 \leq i<$ MaxChanCap $\wedge i \in$ Naturals $\}$

MaxNumChanSet $=\{i \mid 0 \leq i \leq$ MaxNumChan $\wedge i \in$ Naturals $\}$

MaxNumProcSet $=\{i \mid 0 \leq i<$ MaxNumProc $\wedge i \in$ Naturals $\}$

The function ValDomMap specifies the values a variable of a given type can take during the execution of a program (Page 93 of [Hol91]).

ValDomMap is a map from BTypes to $2^{\text {Integers }}$ defined as follows:

ValDomMap (bit) $=\{i \mid 0 \leq i \leq 1 \wedge i \in$ Naturals $\}$

ValDomMap (byte) $=\{i \mid 0 \leq i \leq 255 \wedge i \in$ Naturals $\}$

ValDomMap (short $)=\left\{i \mid-2^{15} \leq i \leq 2^{15}-1 \wedge i \in\right.$ Integers $\}$

ValDomMap $($ int $)=\left\{i \mid-2^{31} \leq i \leq 2^{31}-1 \wedge i \in\right.$ Integers $\}$

ValDomMap $($ chan $)=$ MaxNumChanSet

The above definition states, for example, that a variable of type bit, can take only one of the two values 0 or 1 . 
2.4. Labeled Transition Systems. A symbolic labeled transition system is the mathematical representation of the information contained in the body of a process type declaration (i.e., a Promela proctype).

Definition 2.2. A symbolic labeled transition system is a tuple of the form

〈Name, Structure, Start, locals, ChansOwned, Active, Param〉 where

- Name is an element of Vars (the name of the proctype).

- Structure is 〈lstates, Act, $\longmapsto$ where lstates is a finite set of local states and transition relation $\longmapsto \subseteq$ lstates $\times$ Act $\times$ lstates. Act is PLabels $\times$ EModes $\times$ Priorities and its elements will be ranged over by $\alpha, \beta, \cdots$. PLabels is the set of primitive statements, i.e., the union of Exprs,Asgns,SendExprs, RecExprs and RunExprs. EModes is the set of execution modes $\{$ normal, atomic, deterministic $\}$. Priorities is the set of natural numbers.

Informally, Structure encodes the control-flow of the sequential program named Name as a labeled directed graph. The nodes of this graph are the elements of set lstates. Each node represents a distinct local state of Name. Relation $\longmapsto$ represents the labeled edges between the nodes. If $\left\langle n_{1}, \alpha, n_{2}\right\rangle \in \longmapsto$ then it is possible to move to state $n_{2}$ from state $n_{1}$ by executing the primitive statement $\alpha .1$. The label of the edge also contains information about whether the statement $\alpha .1$ is to executed normally, atomically or deterministically. We call this the execution mode, and encode it as $\alpha .2$. Finally, $\alpha .3$ represents the priority of the transition. Priorities will be used to properly encode unless statements. A transition with a lower priority will not be selected for execution if one with strictly higher priority is also executable.

- Start is an element of lstates.

It represents the initial state of the proctype.

- locals is a partial function from the Vars to $\cup\{\operatorname{ValDomMap}(\tau) \mid \tau \in$ BTypes\}.

The partial function locals identifies the local variables of Name. Note that the function locals is partial; given a variable name var, locals(var) is undefined if var is not a local variable of Name. If var is indeed a local variable, then locals (var) is 0 (the default value, it it is uninitialized) or the value to which it is initialized. locals does not contain information about channel variables.

- ChansOwned is a finite sequence of elements from

$($ Vars $\times($ MaxChanCapSet $\cup\{\Omega\}) \times($ Types $\cup\{\Omega\})$.

The entity ChansOwned records the information about the channel variables accessed by this sequential program; specifically, it records the capacity of each channel (the maximum number of messages the individual channel can hold) and the data type of its message field. For an uninitialized variable of type chan these values above are set to $\Omega$.

- Active is an integer which is either 0 or 1.

- Param is a member of Vars. 
This is a single formal parameter that is bound to a value when the process is instantiated.

(End of definition 2.2.)

2.5. Local and Global States. Lstates is the set of local states (i.e., process states). Gstates is the set of global states (i.e., system states). Formally, a global state $\Sigma$ is a pair of the form $\langle X, g\rangle . X$ is a finite set of elements of the form $\langle$ Name, pid, pc, locals, lChansOwned $\rangle$. Each process active in the state $\Sigma$ is represented by some member of $X$ exactly once.

1. Name is an element of Vars.

It is the name of the proctype corresponding to this process. Note that there could be more than one active process corresponding to the same proctype.

2. pid belongs to MaxNumProcSet.

It is the unique identifier corresponding to an active process. In other words, if $x$ and $x^{\prime}$ are two distinct members of $X$ then $x .2 \neq x^{\prime} .2$. Thus any member of $X$ can be uniquely identified by pid. Thus we denote $X: i$ to denote that particular element of $x$ of $X$ such that $x$.pid $=i$.

3. $p c$ belongs to Lstates

It is the program counter of the active process; it points to some local state.

4. locals is a partial function from Vars to $\cup\{\operatorname{ValDomMap}(\tau) \mid \tau \in$ BTypes \}.

It is the set of local variables of the active process.

5. lChansOwned is a finite sequence of elements from

$($ Vars $\times($ MaxChanCapSet $\cup\{\Omega\}) \times($ Types $\cup\{\Omega\})$.

It is the list of channels owned by this active process.

The second of component $g$ of the global state $\Sigma$ is a tuple of the kind 〈globals, gChansOwned, NrProcs, Handshake, Exclusive, ChIdMap,

ChContMap, timeout, dstep $\rangle$ where

1. globals is a partial function from Vars to $\cup\{\operatorname{ValDomMap}(\tau) \mid \tau \in$ BTypes $\}$

It is the set of global variables.

2. gChansOwned is a finite sequence of elements from

$($ Vars $\times($ MaxChanCapSet $\cup\{\Omega\}) \times($ Types $\cup\{\Omega\})$.

It is the list of globally declared channels.

3. NrProcs is an element of MaxNumProcSet.

$\mathrm{NrProcs}+1$ is the number of processes currently active at this global state. It cannot exceed MaxNumProc.

4. Handshake is either an empty set or an element of MaxNumProcSet $\times$ MaxNumChanSet $\times$ Consts.

If Handshake is not empty it means that there is a synchronous communication (a rendezvous handshake) in progress; the active process with process-id Handshake.1 is sending the value Handshake.3 over the channel Handshake.2.

5. Exclusive is either empty or an element of MaxNumProcSet.

If Exclusive is non-empty the process with process-id Exclusive is executing atomically or deterministically, and cannot be interrupted by other process executions. 
6. ChIdMap is a partial function from (MaxNumProcSet $\cup\{-1\}) \times$ Vars to MaxNumChanSet.

This partial function assign an unique channel-id to every every active channel; given a process-id and a variable name as arguments this function provides the channel-id. Global channels are assigned process-id -1. If the variable name is not a channel name for that process-id then the function is undefined on these arguments.

7. ChContMap is a partial function from MaxNumChanSet to

$$
\bigcup\left\{[\cup\{\operatorname{ValDomMap}(\tau) \mid \tau \in \text { Types }\}]^{i} \mid 0 \leq i \leq \operatorname{MaxChanCap}\right\}
$$

Given a channel-id, this maps provides the current contents of that channel.

8. timeout and dstep are integers which take value either 0 or 1 .

If all processes in the system are blocked (i.e., there is no executable statement) when the value of timeout equals 0 , then the value of changes to 1 for one execution step, and returns to its default value 0 . If dstep equals 1 , some process is executing deterministically.

2.6. Auxiliary Functions. This subsection is devoted a list of auxiliary functions that are used for querying and updating global states.

2.6.1. Characteristic Functions. When the same identifier is used to define both a global variable and a local variable, the usual scoping rules determine which variable is intended. The following functions can be used to resolve these issues.

Islchan is a function defined from Vars $\times$ MaxNumProcSet $\times$ Gstates to the set of truth values $\{t t, f f\}$ as follows. Islchan(var,pid, $\langle X, g\rangle)$ is $t t$ if there exists an $x$ in $(X:$ pid).lChansOwned such that $x .1$ equals var and ff otherwise. Intuitively, Islchan (var, pid, $\langle X, g\rangle)$ is true iff in the global state $\langle X, g\rangle$, var is a local channel owned by a process whose process-id is pid.

Islnumvar is a function defined from Vars $\times$ MaxNumProcSet $\times$ Gstates to the set of truth values $\{t t, f f\}$ as follows. Islnumvar $(v a r, p i d,\langle X, g\rangle)$ is $t t$ if $(X: p i d)$.locals $(v a r)$ is well defined and $f f$ otherwise.

Isgchan is a partial function defined from Vars $\times$ MaxNumProcSet $\times$ Gstates to the set of truth values $\{t t, f f\}$ as follows. Isgchan(var,pid, $\langle X, g\rangle)$ is $t t$ if Islnumvar (var,pid, $(X: p i d)$.) as well Islchan(var, pid, $\langle X, g\rangle)$ are ff and there exists an $x$ in g.gChansOwned such that $x .1$ equals var and $f f$ otherwise. Intuitively, a variable is a global channel variable only if there is no local numeric or local channel variable of the same name.

Ischan is a function defined from Vars $\times$ MaxNumProcSet $\times$ Gstates to the set of truth values $\{t t, f f\}$ as follows. Ischan $(v a r, p i d, \Sigma)$ is $t t$ if either $\operatorname{Isgchan}(v a r, p i d, \Sigma)$ is $t t$ or Islchan (var,pid, $\Sigma)$ is $t t$ and ff otherwise.

Isgnumvar is a function defined from Vars $\times$ MaxNumProcSet $\times$ Gstates to the set of truth values $\{t t, f f\}$ as follows. Isgnumvar (var,pid, $\langle X, g\rangle)$ is $t t$ if g.globals(var) is well defined and Islnumvar(var) is ff and Islchan(var) is ff. Otherwise it is ff.

Isnumvar is a partial function defined from Vars $\times$ MaxNumProcSet $\times$ Gstates to the set of truth values $\{t t, f f\}$ as follows. Isnumvar (var,pid, $\Sigma)$ is $t t$ if either Isgnumvar (var,pid, $\Sigma)$ is $t t$ or Islnumvar $(v a r, p i d, \Sigma)$ is $t t$ and ff otherwise. 
2.6.2. Other Auxiliary Functions. We define some more auxiliary functions that depend on the characteristic functions. The function ChanId, given a variable name and a process-id provides the unique channel-id associated with it if it is indeed a channel. Its definition makes use of the characteristic functions to ensure the proper scoping rule is enforced. Similary the function RightVal, given a variable and a process-id provides the right value associated with the variable; if it is a channel variable then its channel-id is returned.

ChanId is a partial function defined from Vars $\times$ MaxNumProcSet $\times$ Gstates to the set (MaxNumChanSet $\cup \Omega)$ as follows. ChanId(var, pid, $\Sigma)$ is $\Omega$ if Ischan (var, pid, $\Sigma$ ) is ff. If Islchan (var, pid, $\Sigma$ ) is tt then ChanId (var,pid, $\Sigma$ ) is $\operatorname{ChIdMap}(\langle$ pid, var $\rangle)$. If $\operatorname{Isgchan}(v a r, p i d, \Sigma)$ is $t t$ then $\operatorname{ChanId}(v a r, p i d, \Sigma)$ is $\operatorname{ChIdMap}(\langle-1, v a r\rangle)$.

RightVal is a partial function defined from Vars $\times$ MaxNumProcSet $\times$ Gstates to the set Integers as follows.

- If Islchan (var,pid, $\Sigma)$ is $t t$ then RightVal(var,pid, $\Sigma)$ is ChanId(var, pid, $\Sigma)$.

- If Islnumvar (var, pid, $\Sigma$ ) is $t t$ then RightVal(var,pid, $\Sigma)$ is locals(var).

- If Isgnumvar (var,pid, $\Sigma)$ is $t t$ then RightVal $(v a r, p i d, \Sigma)$ is globals(var).

- If $I \operatorname{sgchan}(v a r, p i d, \Sigma)$ is $t t$ then RightVal(var, pid, $\Sigma)$ is ChanId(var, $-1, \Sigma)$.

capacity is a partial function defined from Vars $\times$ MaxNumProcSet $\times$ Gstates to the set MaxChanCapSet as follows. If Islchan(var,pid, $\Sigma$ ) is $t t$ then capacity $(v a r, p i d, \Sigma)$ is $x .2$ where $x$ is a element of the sequence lChansOwned such that $x .1$ is same as var. If Isgchan (var,pid, $\Sigma)$ is $t t$ then capacity $($ var, pid, $\Sigma)$ is $x .2$ where $x$ is a element of the sequence $g$ ChansOwned such that $x .1$ is same as var.

compatible $\left(\operatorname{var}_{1}, \operatorname{var}_{2}, p i d, \Sigma\right)$ is $t t$ if $\operatorname{RightVal}\left(\operatorname{var}_{1}, p i d, \Sigma\right)$ is same as RightVal $\left(\operatorname{var}_{2}\right.$, pid,,$\left.\Sigma\right)$ and $f f$ otherwise. compatible(const, var, pid, $\left.\Sigma\right)$ is $t t$ if RightVal $\left(\right.$ var $_{1}$, pid, $\left.\Sigma\right)=$ const and ff otherwise. compatible $\left(\right.$ const $_{1}$, const $\left._{2}\right)$ is $t t$ if const $_{1}=$ const $_{2}$. Synchronous communication can happen only if there is some compatibility between the sender and the receiver; the above function formalizes this relationship.

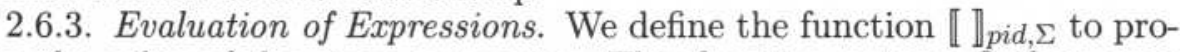
vides the value of the given expression. This function returns $\Omega$ if an error occurs during the evaluation.

The function $\llbracket \rrbracket_{p i d, \Sigma}$ from Exprs to Integers $\cup\{\Omega\}$ is defined as follows. Let $\Sigma=\langle X, g\rangle$. If there is no element $x$ of $X$ with $x .2$ equals pid then $\llbracket \rrbracket_{p i d, \Sigma}$ is a constant function with value $\Omega$. Otherwise let $x$ be the element $\langle$ Name, pid, pc, locals, lChansOwned $\rangle$. Now 【expr $\rrbracket_{p i d, \Sigma}$ is given by structural induction below. For readability, we omit the subscripts for 【】 below.

- If expr $\equiv$ const then $\llbracket$ expr $\rrbracket=$ const.

- If expr $\equiv$ timeout then $\llbracket$ expr $\rrbracket=g . t i m e o u t$.

- If expr $\equiv \operatorname{var}$ then $\llbracket \exp r \rrbracket=$ RightVal(var,pid, $\Sigma)$.

- If expr $=$ LENvar and Ischan $(v a r, p i d, \Sigma)=f f$ then $\llbracket \exp r \rrbracket=\Omega$.

- If $\operatorname{expr}=\mathrm{LEN} v a r$ and $\operatorname{Ischan}(v a r, p i d, \Sigma)=t t$ and $\llbracket v a r \rrbracket=0$ then $\llbracket \exp \eta=\Omega$.

- If expr $=$ LENvar and Ischan (var,pid, $\Sigma)=t t$ and $\llbracket v a r \rrbracket \neq 0$ and ChContMap $(\llbracket v a r \rrbracket)$ is undefined then $\llbracket \exp r \rrbracket=\Omega$ 
- If expr = LENvar and Ischan(var,pid, $\Sigma)=t t$ and $\llbracket v a r \rrbracket \neq 0$ and $\operatorname{ChContMap}(\llbracket v a r \rrbracket)$ is well defined then $\llbracket \exp r \rrbracket=|\operatorname{ChContMap}(\llbracket v a r \rrbracket)|$.

- If $\exp r=\operatorname{var}$ ? [convar $]$ and $($ Ischan $(v a r)=f f$ or $\llbracket \mathrm{LEN} v a r \rrbracket=\Omega)$ then $\llbracket \exp r \rrbracket=\Omega$

- If $($ expr $=$ var? [convar $]$ and $\llbracket \mathrm{LEN} v a r \rrbracket=0$ then $\llbracket e x p r \rrbracket=0$

- If expr $=$ var? [convar] and Ischan $($ var $)=t t$ and $\llbracket$ LENvar $\notin\{0, \Omega\}$ and

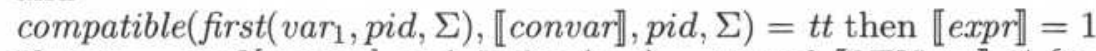

- If $\exp r=\operatorname{var}$ ? $[$ convar $]$ and Ischan $(v a r)=t t$ and $\llbracket$ LENvar $\rrbracket \notin\{0, \Omega\}$ and compatible $\left(\right.$ first $\left(\right.$ var $_{1}$, pid,$\left.\left.\Sigma\right), \llbracket \operatorname{convar} \rrbracket, p i d, \Sigma\right)=f f$ then $\llbracket \exp r \rrbracket=0$

- If $\exp r=\left(\exp r_{1}\right)$ then $\llbracket \exp r \rrbracket=\llbracket \exp r_{1} \rrbracket$

- If $\exp r=\mathrm{uop}$ exp $r_{1}$ then $\llbracket \exp r \rrbracket=\llbracket u o p \rrbracket\left(\llbracket \exp r_{1} \rrbracket\right)$.

If $\exp r=\exp r_{1}$ bop $\exp r_{2}$ then $\llbracket \exp r \rrbracket=\llbracket b o p \rrbracket\left(\llbracket e x p r_{1} \rrbracket, \llbracket e x p r_{2} \rrbracket\right)$.

first is a partial function defined from Vars $\times$ MaxNumProcSet $\times$ Gstates to the set Integers as follows. first (var, pid, $\Sigma)=$ ChContMap $(\llbracket v a r \rrbracket) .1 .2$.

2.6.4. Execution Errors, Failure. As an example of an execution error, consider the statement $x=5$, where $x$ is channel variable. The function WillFail formally describes these situations.

WillFail is a partial function defined from PLabels $\times$ MaxNumProcSet $\times$ Gstates to the set of truth values $\{t t, f f\}$ as follows.

- WillFail (expr, pid, $\Sigma$ ) is $t t$ if $\llbracket \exp \rrbracket \rrbracket$ is $\Omega$ and ff otherwise.

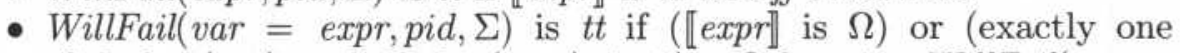
of Ischan(var) and Ischan(expr) is $t t)$. Otherwise WillFail(var = expr, pid, $\Sigma)$ is ff.

- WillFail(var!expr, pid, $\Sigma$ ) is $t t$ if $\llbracket \exp r \rrbracket$ is $\Omega$ or (Ischan(var) is ff) or (Ischan (var) is $t t \wedge$ ChContMap (ChanId(var, pid, $\Sigma)$ ) is undefined).

Otherwise WillFail(var!expr, pid, $\Sigma$ ) is ff.

- WillFail(var? convar, pid, $\Sigma)$ is $t t$ if $(\llbracket$ convar $\rrbracket$ is $\Omega$ ) or (Ischan(var) is $f f)$ or (Ischan(var) is tt and ChanId(var,pid, $\Sigma)=0$ )

or (Ischan (var) is $t t \wedge$ ChContMap (ChanId(var, pid, $\Sigma))$ is undefined. Otherwise WillFail(var? convar, pid, $\Sigma$ ) is ff.

2.6.5. Channel Manipulation Functions. When a new process is created, all the channels owned by it are entered in the ChIdMap. Similary when a process terminates, it local channels are removed from the ChIdMap. The functions defined in this subsubsection accomplish this task.

Definition 2.3. Incorp(ChIdMap, ChansOwned, pid) is defined as a partial function $f$ from the set (MaxNumProcSet $\cup\{-1\}$ ) $\times$ Vars to the set MaxNumChanSet. $f$ has the same value on those elements at which ChIdMap is well defined. Further if $\langle$ var, $\Omega, \Omega\rangle$ is a member of ChansOwned then $f(\langle$ pid, var $\rangle)$ is defined as 0 . Let $c_{1} \cdots c_{k}$ be the sequence ChansOwned with no elments of the form $\langle v a r, \Omega, \Omega\rangle$. Let $m$ be the maximum value taken by ChIdMap. Then $f\left(\right.$ pid, $\left.c_{i} .1\right)$ is defined to be $m+i$ for $1 \leq i \leq k$.

The function Incorp augments the information in the ChIdMap with that present in ChansOwned to produce another function that is of the same type as ChIdMap.

DEFinition 2.4. IncorpCont(ChContMap, ChIdMap, ChansOwned, pid) is a partial function $f$ that has the same domain and codomain as that 
of ChContMap. $f(x)$ is defined as an empty sequence if there exists a $c \in$ ChansOwned such that ChIdMap (pid, $c)=x$ and $x \neq 0$. At other arguments $f$ and ChContMap are identical.

The following two functions remove information from the ChIdMap and ChContMap.

DeFINITION 2.5. RetractId(ChIdMap, ChansOwned, pid) is a partial function $f$ from the set (MaxNumProcSet $\cup\{-1\}) \times$ Vars to MaxNumChanSet. $f($ pid, $c)$ is undefined if there exists a $x \in$ ChansOwned such that $x .1=c$. At other arguments, $f$ and ChIdMap are identical.

Definition 2.6. RetractCont (ChContMap, ChIdMap, ChansOwned, pid) is a partial function $f$ that has the same domain and codomain as that of ChContMap. $f(x)$ is undefined if there exists a $c \in$ ChansOwned such that ChIdMap $($ pid, $c)=x$. At other arguments $f$ and ChContMap are identical.

\section{Preconditions}

We refer to the directed labeled edges of a symbolic transition system as transitions. This section gives the definition of a transition and a current transition. A current transition is executable in a global state only if certain conditions are satisfied. This section formalizes these notions.

DEFINITION 3.1. If $S$ is a symbolic labeled transition system with $S .1=$ Name and S.2.3 contains $\langle s, \alpha, t\rangle$ then $\langle$ Name, $s, \alpha, t\rangle$ will be called a transition.

Informally, a transition is current in a global state $\Sigma$ if the program counter of some active sequential program points to the source state of the transition.

Definition 3.2. A current transition of a state $\Sigma=\langle X, g\rangle$ is the tuple $\langle$ pid, Name, $s, \alpha, t\rangle$ if the following hold:

1. $\langle$ Name, $s, \alpha, t\rangle$ is a transition.

2. $\langle$ Name, pid, s, locals, lChansOwned $\rangle$ is a member of $X$ for some locals and lChansOwned.

Next, we define when a current transition is said to be executable.

Definition 3.3. A current transition $\langle$ Name, pid, $s, \alpha, t\rangle$ is executable in the state $\Sigma=\langle X, g\rangle$ if all of the following 10 conditions hold.

1. g. Exclusive $=\{\}$ or g. Exclusive $=\{$ pid $\}$.

Intuitively, this condition states that in order for a current transition to be executable no other process may have exclusive control.

2. WillFail $(\alpha$. label, pid, $\Sigma)=t t$

$\Longrightarrow$ g. Handshake $=\{\}$.

If a synchronous communication is in progress, a current transition is not permitted to execute and cause an error.

3. $(\alpha$. label $\equiv$ expr $) \wedge$ WillFail $(\alpha$. label, pid,$\Sigma)=f f$

$\Longrightarrow$.

g.Handshake $=\{\} \wedge(\llbracket \exp r \rrbracket \Sigma$, pid $\neq 0)$.

An expression is executable if it value is nonzero and if no synchronous communication is in progress. 
4. $\alpha$. label $\equiv \operatorname{var} ! \operatorname{expr} \wedge$ WillFail $(\alpha . l a b e l$, pid,$\Sigma)=f f \wedge \operatorname{capacity}($ var, pid,,$\Sigma) \neq$ 0

$\Longrightarrow$

g. Handshake $=\{\} \wedge \llbracket L E N v a r \rrbracket_{\text {pid }, \Sigma}<\operatorname{capacity}($ var, pid,$\Sigma)$.

The statement that sends a value of the expr to the channel var is executable only if the the channel var is not full. (see Page 99 of [Hol91])

5. $\alpha$. label $\equiv$ var? convar $\wedge$ WillFail $(\alpha$. label, pid, $\Sigma)=f f$

$\wedge$ capacity $($ var, pid, $\Sigma) \neq 0$

$\Longrightarrow$ g.Handshake $=\{\} \wedge \llbracket$ var? $[$ convar $] \rrbracket_{\text {pid }, \Sigma}=1$.

Similarly the statement that extracts a value from the channel var is executable only if the channel var is nonempty (see Page 100 of [Hol91]).

6. $\alpha$. label $\equiv \operatorname{var} ! \operatorname{expr} \wedge$ WillFail $(\alpha$.label, pid,$\Sigma)=f f \wedge \operatorname{capacity}(\operatorname{var}$, pid,,$\Sigma)=$ 0

$\Longrightarrow$

g. Handshake $=\{\} \wedge$

$\exists$ qid, Name ${ }^{\prime}, s^{\prime}, t^{\prime}, x, \beta:$ qid $\neq$ pid $\wedge \beta$.label $\equiv v a r_{1}^{\prime}$ ? convar $\wedge$

$\llbracket v a r_{1}^{\prime} \rrbracket_{\Sigma, q i d}=\llbracket v a r \rrbracket_{\Sigma, p i d} \wedge\left\langle q i d, N_{a m e}^{\prime}, s^{\prime}, \beta, t^{\prime}\right\rangle$ is a current transition of $\Sigma \wedge$ compatible (convar, \expr $\rrbracket_{g, \text { pid }}$, pid,$\left.\Sigma\right)$.

A synchronous-send statement on a channel var is executable only if there is another distinct process that has synchronous-receive statement on the same channel as a current transition. (see Page 100 of [Hol91])

7. $\alpha$. label $\equiv$ var? convar $\wedge$ WillFail $(\alpha$. label, pid, $\Sigma)=f f \wedge$

$\operatorname{capacity}($ var, pid, $\Sigma)=0$

$\Longrightarrow$

$\exists$ qid, const $:$ qid $\neq$ pid $\wedge$ g.Handshake $=\left\{\left\langle q i d, \llbracket v a r \rrbracket_{p i d, \Sigma}\right.\right.$, const $\left.\rangle\right\}$

$\wedge$ compatible (convar, const, pid, $\Sigma)$.

8. $(\alpha$. label $\equiv$ asgn $) \wedge$ WillFail $(\alpha$. label, pid,,$\Sigma)=f f$

$\Longrightarrow$

g. Handshake $=\{\}$.

9. ( $\alpha$. label $\equiv$ run Name arg $) \wedge$ WillFail $(\alpha$. label, pid,$\Sigma)=f f$

$\Longrightarrow$

g. Handshake $=\{\} \wedge$ g.NrProcs $<$ MaxNumProc -1 .

A new process can be created only if the number of currently active processes does not exceed the preset upper bound.

10. $(\alpha$. label $\equiv$ end $)$

$\Longrightarrow$

g. Handshake $=\{\} \wedge$ pid $=$ g.NrProcs.

Processes can only terminate in the reverse order of their creation (i.e., a process cannot terminate until all its children have terminated first). If processes are issued pid's sequentially starting from zero, then the only process that can terminate is the one that with a pid equal to g.NrProcs. (see Page 96 of [Hol91])

\section{Effects}

In this section we define how each of the primitive statements transforms the current global state when it is executed. 
4.1. Normal Execution Mode. The effect of the execution of a statement depends in part on the "mode" in which it is executed. The following definition presents the effects of statements executed in "normal" mode (i.e., not involving atomic or dstep sequences).

Definition 4.1. If $\langle$ pid, Name, $s, \alpha, t\rangle$ is executable in the state $\Sigma=$ $\langle X, g\rangle$ with $\alpha$.mode $=$ normal then Effect $(\Sigma,\langle$ pid, Name, $s, \alpha, t\rangle)$ is $\Sigma^{\prime}=$ $\left\langle X^{\prime}, g^{\prime}\right\rangle$ where

1. WillFail $(\alpha . l a b e l$, pid, $\Sigma)=t t \Longrightarrow \Sigma^{\prime}=\Omega$

We use the symbol $\Omega$ to denote the global error state. The above condition states that the execution of an error-producing statement will transform any state into the error state.

2. $(\alpha$. label $\equiv$ expr $) \wedge$ WillFail $(\alpha$. label, pid,,$\Sigma)=f f \wedge \llbracket$ exp $r \rrbracket_{p i d, \Sigma} \neq 0$

$\overrightarrow{X^{\prime}}=X[\langle$ Name, pid, $t$, locals, lChansOwned $\rangle /$

$\langle$ Name, pid, s, locals, lChansOwned $\rangle]$

$g^{\prime}=g[$ Exclusive $=\{\}$, dstep $=0]$

The program counter of the process is updated. No exclusive execution control is obtained or preserved by any statement that is executed in normal mode.

3. $(\alpha . l a b e l \equiv v a r=\exp r \wedge$ WillFail $(\alpha . l a b e l, p i d, \Sigma)=f f)$

$\overrightarrow{X^{\prime}}=X[\langle$ Name,pid,t, locals, lChansOwned $\rangle /$

$\langle$ Name, pid, s, locals', lChansOwned $\rangle]$

$g^{\prime}=g[$ Exclusive $=\{\}$, dstep $=0$, globals $/$ globals,

ChIdMap'/ChIdMap] where

- islnumvar $($ var $)=t t$

$\Longrightarrow\left(\right.$ locals $^{\prime}=$ locals $\left[\right.$ var $=\llbracket$ expr $\left.\rrbracket_{\text {pid, }, \Sigma}\right]$, globals $^{\prime}=$ globals,

ChIdMap $=$ ChIdMap)

- isgnumvar $($ var $)=t t$

$\Longrightarrow$ (globals ${ }^{\prime}=$ globals $\left[\right.$ var $=\llbracket$ expr $\left.\rrbracket_{\text {pid }, \Sigma}\right]$, locals $^{\prime}=$ locals,

ChIdMap' $=$ ChIdMap)

- $i s c h a n(v a r)=t t$

$\Longrightarrow$ locals $^{\prime}=$ locals, globals ${ }^{\prime}=$ globals,

ChIdMap ${ }^{\prime}=\operatorname{ChIdMap}\left[\operatorname{ChanId}(\right.$ var, pid,$\left.\left.\Sigma)=\llbracket \operatorname{expr} \rrbracket_{p i d, \Sigma}\right]\right)$

The effect of this statement is to assign the value of the expression to the variable. If the variable is a channel variable then the ChIdMap is updated. The scope rules determine if the variable is local or global.

4. $(\alpha . l a b e l \equiv \operatorname{var} !$ expr $\wedge$ capacity $($ var $, p i d, \Sigma) \neq 0 \wedge$ WillFail $(\alpha . l a b e l, p i d, \Sigma)=$ ff)

$\overrightarrow{X^{\prime}}=X[\langle$ Name, pid, $t$, locals, lChansOwned $\rangle /$

$\langle$ Name, pid, s, locals, lChansOwned $\rangle]$

$g^{\prime}=g[$ Exclusive $=\{\}$, dstep $=0$, ChContMap' $/$ ChContMap $]$ where

ChContMap' is given by ChContMap $[$ ChIdMap $($ pid, var,$\Sigma)=$ ChContMap $(\operatorname{ChIdMap}($ pid, var,,$\Sigma)) \circ \llbracket \exp r \rrbracket_{p i d, \Sigma} \rrbracket$

To understand the above condition, recall the meaning of the notation $f[x=$ $y$ ] from Subsection 2.1; the value of the expression is appended at the end of the contents of the channel var. (See Page 98 of [Hol91]). 
5. ( .label $\equiv$ var? const $\wedge$ capacity $($ var, pid,$\Sigma) \neq 0$

$\wedge$ WillFail $(\alpha . l a b e l$, pid,,$\Sigma)=f f)$

$\overrightarrow{X^{\prime}}=X[\langle$ Name, pid, $t$, locals, lChansOwned $\rangle /$

$\langle$ Name, pid, s, locals, lChansOwned $\rangle]$

$g^{\prime}=g[$ Exclusive $=\{\}$, dstep $=0$, ChContMap' $/$ ChContMap $]$ where

ChContMap' is given by ChContMap [ChIdMap $($ pid, var, $\Sigma)=$

$\operatorname{cdr}(\operatorname{ChContMap}(\operatorname{ChIdMap}($ pid, $v a r, \Sigma)))]$

Since this transition is executable it is necessarily the case that the head of the channel var is const.

6. $\left(\alpha . l_{\text {label }} \equiv \operatorname{var}_{1}\right.$ ?var $_{2} \wedge$ capacity $\left(\right.$ var $_{1}$, pid,$\left.\Sigma\right) \neq 0$

$\wedge$ WillFail $(\alpha . l a b e l, p i d, \Sigma)=f f)$

$\Longrightarrow \overrightarrow{X^{\prime}}=X[\langle$ Name, pid, $t$, locals, lChansOwned $\rangle /$

$\langle$ Name, pid, s, locals', lChansOwned $\rangle]$

$g^{\prime}=g[$ Exclusive $=\{\}$, globals $/$ globals, ChIdMap $/$ ChIdMap,

dstep $=0$, ChContMap $/$ ChContMap $]$ where

- islnumvar $\left(\operatorname{var}_{1}\right)=t t$

$\Longrightarrow\left(\right.$ locals $^{\prime}=$ locals $\left[\right.$ var $_{2}=$ first $\left(\operatorname{var}_{1}\right.$, pid,$\left.\left.\Sigma\right)\right]$,

globals $^{\prime}=$ globals, ChIdMap $=$ ChIdMap $)$

- $i \operatorname{schan}($ var $)=t t$

$\Longrightarrow\left(\right.$ locals $^{\prime}=$ locals, globals ${ }^{\prime}=$ globals,

ChIdMap' $=$ ChIdMap $\left[\right.$ ChanId $\left.\left.\left(\operatorname{var}_{2}, \operatorname{pid}, \Sigma\right)=\operatorname{first}\left(\operatorname{var}_{1}, \operatorname{pid}, \Sigma\right)\right]\right)$

- isgnumvar $($ var $)=t t$

$\Longrightarrow\left(\right.$ globals $^{\prime}=$ globals $\left[\right.$ var $_{2}=$ first $\left(\right.$ var $_{1}$, pid,$\left.\left.\Sigma\right)\right]$, locals $^{\prime}=$ locals, ChIdMap' $=$ ChIdMap)

- ChContMap ${ }^{\prime}=$

ChContMap $[$ ChIdMap $($ pid, var, $\Sigma)=$

$\operatorname{cdr}(\operatorname{ChContMap}(\operatorname{ChIdMap}($ pid, $\operatorname{var}, \Sigma)))]$

The value at the head of the channel $\operatorname{var}_{1}$ is removed and assigned to the value $v r_{2}$, just like in a normal PROMELA assignment statement.

7. $(\alpha . l a b e l \equiv \operatorname{var} ! \exp r \wedge \operatorname{capacity}($ var, pid,,$\Sigma)=0 \wedge$ WillFail $(\alpha . l a b e l, p i d, \Sigma)=$ $f f)$

$\overrightarrow{X^{\prime}}=X[\langle$ Name, pid,, locals, lChansOwned $\rangle /$

$\langle$ Name, pid, s, locals, lChansOwned $\rangle]$

$g^{\prime}=g[$ Exclusive $=\{\}$, dstep $=0$,

Handshake $=\left\{\left\langle\right.\right.$ pid, ChanId $($ var, pid,$\left.\left.\left.\Sigma), \llbracket \operatorname{expr} \rrbracket_{\text {pid }, \Sigma}\right\rangle\right\}\right]$

The effect of a synchronous-send statement is to store the following values in the mathematical structure Handshake: the pid of the sender process, the channel-id of the synchronous channel and the value of the expression being sent. This signifies the initiation of a synchronous (rendezvous hand-shake) communication.

8. $(\alpha$. label $\equiv$ var? const $\wedge$ capacity $($ var, pid,$\Sigma)=0 \wedge$ WillFail $(\alpha . l a b e l, p i d, \Sigma)=$ $f f \wedge$ Handshake $=\left\{\left\langle q i d, \llbracket\right.\right.$ var $\rrbracket_{\text {pid }, \Sigma}$, const $\left.\left.\left.^{\prime}\right\rangle\right\}\right)$

\footnotetext{
$\overrightarrow{X^{\prime}}=X[\langle$ Name, pid, t, locals, lChansOwned $\rangle /$
} 
$\langle$ Name, pid, s, locals, lChansOwned $\rangle]$

$g^{\prime}=g[$ Exclusive $=\{\}$, dstep $=0$, Handshake $=\{\}]$

Since this transition is executable, it is necessarily the case that qid $\neq$ pid and const $^{\prime}=$ const. One of the effect of this transition is to set structure Handshake to \{\} , which signifies the completion of the synchronous communication.

9. $\left(\alpha\right.$. label $\equiv v a r_{1}$ ? var $_{2} \wedge$ capacity $\left(\right.$ var $\left._{1}, p i d, \Sigma\right)=0$

$\wedge$ WillFail $(\alpha . l a b e l, p i d, \Sigma)=f f)$

$\wedge$ Handshake $=\left\{\left\langle\right.\right.$ qid,$\llbracket v a r_{1} \rrbracket_{\text {pid }, \Sigma}$, const $\left.\rangle\right\}$

$\overrightarrow{X^{\prime}}=X[\langle$ Name, pid, $t$, locals, lChansOwned $\rangle /$

$\langle$ Name, pid, s, locals', lChansOwned $\rangle]$

$g^{\prime}=g[$ Exclusive $=\{\}$, globals $/$ globals, dstep $=0$, ChIdMap' $/$ ChIdMap $]$ where

- islnumvar $\left(\operatorname{var}_{2}\right)=t t$

$\Longrightarrow\left(\right.$ locals $^{\prime}=$ locals $\left[\right.$ var $_{2}=$ const $]$, globals ${ }^{\prime}=$ globals, ChIdMap ${ }^{\prime}=$ ChIdMap)

- $i \operatorname{schan}\left(\right.$ var $\left._{2}\right)=t t$

$\Longrightarrow$ locals $^{\prime}=$ locals, globals ${ }^{\prime}=$ globals,

ChIdMap ${ }^{\prime}=$ ChIdMap $\left[\right.$ ChanId $\left(\right.$ var $_{2}$, pid,$\left.\Sigma\right)=$ const $\left.]\right)$

- isgnumvar $\left(\right.$ var $\left._{2}\right)=t t$

$\Longrightarrow\left(\right.$ globals ${ }^{\prime}=$ globals $\left[\right.$ var $_{2}=$ const $]$, locals $^{\prime}=$ locals, ChIdMap ${ }^{\prime}=$ ChIdMap)

The effect of this statement is to assign the value in Handshake to the variable $v r_{2}$ and to make the Handshake equal to \{\} signifying the termination of the synchronous communication.

10. $(\alpha$. label $\equiv$ run Name 1 arg $) \wedge$ WillFail $(\alpha . l a b e l, p i d, \Sigma)=f f) \wedge$

$\left\langle\right.$ Name $_{1}$, Structure $_{1}$, Start $_{1}$, locals $_{1}$, ChansOwned $_{1}$, Active $_{1}$, Param $\left._{1}\right\rangle$

is a symbolic transition system $\Longrightarrow$

$X^{\prime \prime}=X[\langle$ Name, pid,, locals, lChansOwned $\rangle /$

$\langle$ Name, pid, s, locals, lChansOwned $\rangle]$

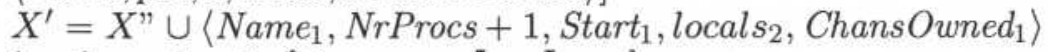

locals $_{2}=$ locals $_{1}\left[\right.$ Param $_{1}=\llbracket$ arg $\left.\rrbracket_{\text {pid, }, \Sigma}\right]$

$g^{\prime}=g[$ Exclusive $=\{\}$, NrProcs $=$ NrProcs +1, dstep $=0$,

Incorp (ChIdMap, ChansOwned 1, NrProcs +1$) /$ ChIdMap,

IncorpCont (ChContMap, ChIdMap, lChansOwned 1 , NrProcs + 1)/

ChContMap]

The effect of this statement is to create a new process with proctype Name.

The smallest unassigned nonnegative integer is assigned as its process-id; this value equals the old value of NrProcs, from before the statement is executed. The formal parameter is instantiated with the value of the actual parameter. ChIdMap and ChContMap are updated accordingly.

11. $(\alpha$. label $\equiv$ end $\wedge$ WillFail $(\alpha$. label, pid,$\Sigma)=f f)$

$\overrightarrow{X^{\prime}}=X \backslash\{\langle$ Name, pid, $t$, locals, lChansOwned $\rangle\}$

$g^{\prime}=g[$ RetractId (ChIdMap, lChansOwned, pid)/ChIdMap,

RetractCont(ChContMap, ChIdMap, lChansOwned, pid)/ChContMap, dstep $=0$, NrProcs $=$ NrProcs -1$]$ 
4.2. Atomic and Deterministic Execution Mode. Generally, when a process executes a primitive statement in atomic or deterministic mode it retains the exclusive right to execute also the next statement (it "retains control"). Otherwise, the effect of the execution of statements in these two modes is same as that in normal mode. To indicate this, in the following definition we set Exclusive $=\{$ pid $\}$ besides the other conditions that are presented in the Definition 4.1. There is only one statement that when executed in atomic mode does not retain control: the synchronous send statement. Furthermore, it is an error to execute synchronous-send and synchronousreceive in deterministic mode.

The parts of the following definition that differ from the corresponding parts in Definition 4.1 are indicated in bold.

Definition 4.2. If $\langle$ pid, Name, $s, \alpha, t\rangle$ is executable in the state $\Sigma=$ $\langle X, g\rangle$ with $\alpha$. mode $\in\{$ atomic, deterministic $\}$ then

Effect $(\Sigma,\langle$ pid, Name, $s, \alpha, t\rangle)$ is $\Sigma^{\prime}=\left\langle X^{\prime}, g^{\prime}\right\rangle$ where

1. WillFail $(\alpha$. label, pid, $\Sigma)=t t \Longrightarrow \Sigma^{\prime}=\Omega$.

2. $(\alpha$. label $\equiv$ expr $) \wedge$ WillFail $(\alpha$.label, pid, $\Sigma)=f f \wedge \llbracket$ exp $r \rrbracket_{\text {pid }, \Sigma} \neq 0$

$\Longrightarrow$

$\overrightarrow{X^{\prime}}=X[\langle$ Name, pid, $t$, locals, lChansOwned $\rangle /$

$\langle$ Name, pid, s, locals, lChansOwned $\rangle]$

$g^{\prime}=g\left[\right.$ Exclusive $=\{$ pid $\}$, dstep $=$ dstep $\left.^{\prime}\right]$

dstep $^{\prime}=1$ if $\alpha$. label $=$ deterministic and 0 otherwise.

3. $(\alpha$. label $\equiv v a r=\exp r \wedge \operatorname{WillFail}(\alpha$. label, pid,$\Sigma)=f f)$

$\Longrightarrow$

$\overrightarrow{X^{\prime}}=X[\langle$ Name, pid, $t$, locals, lChansOwned $\rangle /$

$\left\langle\right.$ Name, pid, s, locals' ${ }^{\prime}$ lChansOwned $\left.\rangle\right]$

$g^{\prime}=g[$ Exclusive $=\{$ pid $\}$, globals $/$ globals, ChIdMap $/$ ChIdMap, dstep' / dstep]

where dstep $^{\prime}=1$ if $\alpha$. label $=$ deterministic and 0 otherwise.

- islnumvar $(\operatorname{var})=t t$

$\Longrightarrow\left(\right.$ locals $^{\prime}=$ locals $\left[\right.$ var $=\llbracket$ expr $\left.\rrbracket_{\text {pid }, \Sigma}\right]$, globals $^{\prime}=$ globals,

ChIdMap' $=$ ChIdMap)

- $i \operatorname{schan}($ var $)=t t$

$\Longrightarrow$ (locals $^{\prime}=$ locals, globals $^{\prime}=$ globals, ChIdMap'

$=C h I d M a p\left[C h a n I d\left(v a r, p i d, \Sigma=\llbracket \exp r \rrbracket_{p i d, \Sigma}\right]\right)$

- isgnumvar $($ var $)=t t$

$\Longrightarrow\left(\right.$ globals $^{\prime}=$ globals $\left[\right.$ var $=\llbracket$ expr $\left.\rrbracket_{\text {pid }, \Sigma}\right]$, locals $^{\prime}=$ locals, ChIdMap' $=$ ChIdMap)

4. $(\alpha$. label $\equiv \operatorname{var!} \exp r \wedge$ capacity $($ var, pid,$\Sigma) \neq 0 \wedge$ WillFail $(\alpha . l a b e l$, pid,$\Sigma)=$ ff)

$X^{\prime}=X[\langle$ Name, pid, $t$, locals, lChansOwned $\rangle /$

$\langle$ Name, pid, s, locals, lChansOwned $\rangle]$

$g^{\prime}=g\left[\right.$ Exclusive $=\{p i d\}$, ChContMap ${ }^{\prime} /$ ChContMap, dstep $/$ dstep $\left.^{\prime}\right]$

dstep $^{\prime}=1$ if $\alpha$.label $=$ deterministic and 0 otherwise. where

ChContMap' ${ }^{\prime}=$ ChContMap $[$ ChIdMap $($ pid, var,$\Sigma)=$

ChContMap $(\operatorname{ChIdMap}($ pid, var,,$\left.\Sigma)) \circ \llbracket \exp r \rrbracket_{p i d, \Sigma}\right]$. 
5. $(\alpha$. label $\equiv$ var?const $\wedge$ capacity $($ var, pid,$\Sigma) \neq 0$

$\wedge \operatorname{WillFail}(\alpha$. label, pid, $\Sigma)=f f)$

$\Longrightarrow \overrightarrow{X^{\prime}}=X[\langle$ Name, pid, $t$, locals, lChansOwned $\rangle /$

$\langle$ Name, pid, s, locals, lChansOwned $\rangle]$

$g^{\prime}=g\left[\right.$ Exclusive $=\{$ pid $\}$, ChContMap' $/$ ChContMap, dstep $/$ dstep $\left.^{\prime}\right]$

where

- dstep' $=1$ if $\alpha$. label $=$ deterministic and 0 otherwise.

- ChContMap' =

ChContMap $[$ ChIdMap $($ pid, var, $\Sigma)=$ $\operatorname{cdr}($ ChContMap $(\operatorname{ChIdMap}($ pid, var, $\Sigma)))]$.

6. $\left(\alpha\right.$. label $\equiv \operatorname{var}_{1}$ ?var $2 \wedge$ capacity $\left(\operatorname{var}_{1}\right.$, pid,$\left.\Sigma\right) \neq 0$

$\wedge \operatorname{WillFail}(\alpha$. label, pid, $\Sigma)=f f)$

$\overrightarrow{X^{\prime}}=X[\langle$ Name, pid, $t$, locals, lChansOwned $\rangle /$

$\langle$ Name, pid, s, locals', lChansOwned $\rangle]$

$g^{\prime}=g[$ Exclusive $=\{$ pid $\}$, globals $/$ globals, ChIdMap $/$ ChIdMap,

ChContMap'/ChContMap, dstep/dstep'] where

- dstep' - $^{\prime}$ if $\alpha$. label $=$ deterministic and 0 otherwise.

- $i \operatorname{sinumvar}\left(\operatorname{var}_{1}\right)=t t$

$\Longrightarrow\left(\right.$ locals $^{\prime}=$ locals $\left[\right.$ var $_{2}=$ first $\left(\right.$ var $_{1}$, pid,,$\left.\left.\Sigma\right)\right]$,

globals ${ }^{\prime}=$ globals, $C h I d M a p^{\prime}=$ ChIdMap)

- $i \operatorname{schan}($ var $)=t t$

$\Longrightarrow$ locals $^{\prime}=$ locals, globals $^{\prime}=$ globals,

ChIdMap' $=$ ChIdMap $\left[\right.$ ChanId $\left(\right.$ var $_{2}$, pid,,$\left.\Sigma\right)=$ first $\left(\operatorname{var}_{1}\right.$, pid,,$\left.\left.\Sigma\right)\right]$

- isgnumvar $($ var $)=t t$

$\left(\right.$ globals $^{\prime}=$ globals $\left[\right.$ var $_{2}=$ first $\left(\right.$ var $_{1}$, pid,$\left.\left.\Sigma\right)\right]$, locals $^{\prime}=$ locals,

ChIdMap' = ChIdMap)

- ChContMap' = ChContMap $[$ ChIdMap (pid, var,$\Sigma)=$ $\operatorname{cdr}(\operatorname{ChContMap}(\operatorname{ChIdMap}($ pid, var, $\Sigma)))]$

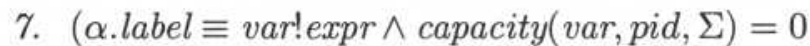

$\wedge \operatorname{WillFail}(\alpha$. label, pid, $\Sigma)=f f)$

$\overrightarrow{X^{\prime}}=X[\langle$ Name, pid, $t$, locals, lChansOwned $\rangle /$

$\langle$ Name, pid, s, locals, lChansOwned $\rangle]$

$g^{\prime}=g[$ Exclusive $=\{\}$,

Handshake $=\left\{\left\langle\right.\right.$ pid, ChanId $($ var, pid,$\Sigma), \llbracket$ exp $\left.\left.\left.r \rrbracket_{\text {pid }, \Sigma}\right\rangle\right\}\right]$

Note that the execution of a synchronous send statement in atomic mode causes the process to lose the right to execute the next statement (that right passes to the receiving process).

8. $(\alpha$. label $\equiv$ var? const $\wedge$ capacity $($ var, pid,$\Sigma)=0$

$\wedge$ WillFail $(\alpha$. label, pid,$\Sigma)=f f)$

$\wedge$ Handshake $=\left\{\left\langle\right.\right.$ qid,$\llbracket$ var $_{1} \rrbracket_{\text {pid }, \Sigma}$, const $\left.\rangle\right\}$

$\overrightarrow{X^{\prime}}=X[\langle$ Name, pid, $t$, locals, lChansOwned $\rangle /$

$\langle$ Name, pid, s, locals, lChansOwned $\rangle]$

$g^{\prime}=g[$ Exclusive $=\{$ pid $\}$, Handshake $=\{\}]$ 
9. ( $\alpha$. label $\equiv \operatorname{var}_{1}$ ? $\operatorname{var}_{2} \wedge$ capacity $\left(\right.$ var $_{1}$, pid,$\left.\Sigma\right)=0$

$\wedge$ WillFail $(\alpha$. label, pid, $\Sigma)=f f)$

$\wedge$ Handshake $=\left\{\left\langle\right.\right.$ qid, $\llbracket$ var $\rrbracket_{1} \rrbracket_{\text {pid }, \Sigma}$, const $\left.\rangle\right\}$

$\Longrightarrow=X[\langle$ Name, pid, $t$, locals, lChansOwned $\rangle /$

$\langle$ Name, pid, s, locals', lChansOwned $\rangle]$

$g^{\prime}=g[$ Exclusive $=\{$ pid $\}$, globals $/$ globals, ChIdMap $/$ ChIdMap $]$ where

- islnumvar $\left(\operatorname{var}_{2}\right)=t t$ $\Longrightarrow\left(\right.$ locals $^{\prime}=$ locals $\left[\right.$ var $_{2}=$ const $]$, globals $^{\prime}=$ globals, ChIdMap' = ChIdMap)

- $i \operatorname{schan}\left(\operatorname{var}_{2}\right)=t t$ $\Longrightarrow$ (locals' $=$ locals, globals $=$ globals, ChIdMap' $=$ ChIdMap $\left[\right.$ ChanId $\left(\right.$ var $_{2}$, pid,,$\left.\Sigma\right)=$ const $]$

- isgnumvar $\left(\operatorname{var}_{2}\right)=t t$

$\Longrightarrow\left(\right.$ globals ${ }^{\prime}=$ globals $\left[\operatorname{var}_{2}=\right.$ const $]$, locals $^{\prime}=$ locals ChIdMap' $=$ ChIdMap)

10. $\left(\alpha\right.$. label $\equiv$ run Name $_{1}$ arg $) \wedge \operatorname{WillFail}(\alpha$. label, pid,$\left.\Sigma)=f f\right) \wedge$

$\left\langle\right.$ Name $_{1}$, Structure $_{1}$, Start $_{1}$, locals $_{1}$, ChansOwned $_{1}$, Active $_{1}$, Param $\left._{1}\right\rangle$

is a symbolic transition system $\Longrightarrow$

$X "=X[\langle$ Name, pid, $t$, locals, lChansOwned $\rangle /$

$\langle$ Name, pid, s, locals, lChansOwned $\rangle]$

$X^{\prime}=X^{\prime \prime} \cup\left\langle\right.$ Name $_{1}$, NrProcs +1, Start $_{1}$, locals $_{2}$, ChansOwned $\left._{1}\right\rangle$

locals $_{2}=$ locals $_{1}\left[\right.$ Param $_{1}=\llbracket$ arg $\left.\rrbracket_{\text {pid, }, \Sigma}\right]$

$g^{\prime}=g[$ Exclusive $=\{$ pid $\}$, NrProcs $=$ NrProcs +1,

Incorp (ChIdMap, ChansOwned 1, NrProcs + 1)/ChIdMap,

IncorpCont (ChContMap, ChIdMap, lChansOwned 1, NrProcs + 1)/

ChContMap, dstep'/dstep]

where dstep $^{\prime}=1$ if $\alpha$. label $=$ deterministic and 0 otherwise.

11. $(\alpha$. label $\equiv$ end $) \wedge$ WillFail $(\alpha$. label, pid, $\Sigma)=f f) \wedge$

$\overrightarrow{X^{\prime}}=X \backslash\{\langle$ Name, pid, $t$, locals, lChansOwned $\rangle\}$

$g^{\prime}=g[$ RetractId (ChIdMap, lChansOwned,pid $) /$ ChIdMap,

RetractCont (ChContMap, ChIdMap, lChansOwned, pid)/ChContMap, NrProcs $=$ NrProcs -1$]$

12. $((\alpha$. label $\equiv$ var! expr $) \vee(\alpha$. label $\equiv$ var? convar $) \wedge$

capacity $($ var, pid,$\Sigma)=0 \wedge \alpha$.mode $=$ deterministic

$\overrightarrow{\Sigma^{\prime}}=\Omega$

Any attempt to perform synchronous communication (rendezvous handshaking) in deterministic mode fails.

\section{Executions}

In this section, we define the executions of a Promela program. So far we have not taken into account the priorities of the transitions into considerations. We define an executable transition to be selectable if there exists no other executable statement with strictly higher priority. The following definition formalizes this statement. 
DEFinition 5.1. A selectable transition of a state $\Sigma=\langle X, g\rangle$ is an executable transition of $\langle$ pid, Name, $s, \alpha, t\rangle$ such that if $\left\langle\right.$ pid, $\left.s, \beta, t^{\prime}\right\rangle$ is any executable transition of $\Sigma$ then $\alpha$.priority $>\beta$.priority.

DEFINITION 5.2. If a transition $t$ is selectable in state $\Sigma$ then $\Sigma \rightarrow 1$ Effect $(\Sigma, t)$.

Selectable transitions almost correspond to actual execution steps of a program, but there are some steps that have not yet been modeled.

If, for instance, a process becomes blocked while it is executing an atomic sequence, then the process loses its exclusive control (Page 98 of [Hol95]). However, if a process becomes blocked when it is executing a dstep sequence (i.e., when it executes in deterministic mode), then it fails. We define $\rightarrow_{2-}$ transitions to augment $\rightarrow_{1}$-transitions with these additional steps.

DEFINITION 5.3. $\rightarrow_{2}$ is the smallest binary relation over Gstates satisfying the following conditions.

1. $\Sigma \rightarrow_{1} \Sigma^{\prime} \Longrightarrow \Sigma \rightarrow_{2} \Sigma^{\prime}$.

2. $\left(\langle X, g\rangle \neq \Omega \wedge \neg\left(\exists\left\langle X^{\prime}, g^{\prime}\right\rangle:\langle X, g\rangle \rightarrow_{1}\left\langle X^{\prime}, g^{\prime}\right\rangle\right) \wedge\langle X, g\rangle[\right.$ g. Exclusive $=$ \{\}$] \rightarrow_{1} \Sigma^{\prime \prime} \wedge$ g.dstep $\left.=0\right) \Longrightarrow\langle X, g\rangle \rightarrow_{2} \Sigma^{\prime \prime}$.

3. $\left(\langle X, g\rangle \neq \Omega \wedge \neg\left(\exists\left\langle X^{\prime}, g^{\prime}\right\rangle:\langle X, g\rangle \rightarrow_{1}\left\langle X^{\prime}, g^{\prime}\right\rangle\right) \wedge\right.$ g.dstep $\left.=1\right) \Longrightarrow$ $\langle X, g\rangle \rightarrow_{2} \Omega$.

We have still not captured all the possible execution steps of a PROMELA program. The predefined variable timeout is set to be 1 when there are no executable statements in the system (Page 125 of [Hol91]). The executions that result by the setting of timeout to 1 , augment $\rightarrow_{2}$-transitions with $\rightarrow_{3}$ transitions, as defined below.

DEFINITION 5.4. $\rightarrow 3$ is the smallest binary relation over Gstates satisfying the following conditions.

1. $\Sigma \rightarrow_{2} \Sigma^{\prime} \Longrightarrow \Sigma \rightarrow_{3} \Sigma^{\prime}$.

2. $\left(\langle X, g\rangle \neq \Omega \wedge \neg\left(\exists\left\langle X^{\prime}, g^{\prime}\right\rangle:\langle X, g\rangle \rightarrow_{1}\left\langle X^{\prime}, g^{\prime}\right\rangle\right) \wedge\langle X, g\rangle[\right.$ g.timeout $=$ $\left.1] \rightarrow_{2}\left\langle X^{\prime \prime}, g^{\prime \prime}\right\rangle\right) \Longrightarrow\langle X, g\rangle \rightarrow_{3}\left\langle X^{\prime \prime}, g^{\prime \prime}\right\rangle\left[g^{\prime \prime}\right.$. timeout $\left.=0\right]$.

In these semantics we have modeled some conceptual single-step executions as multiple-step executions. For example, synchronous communication is modeled in two steps (a send followed by a receive). This resulted in the introduction of an "invisible" system state (the one in which the value is already sent but has not yet been received). Similarly, the intermediate states in a dstep sequence are invisible. The following definition formalizes this.

Definition 5.5. A state $\Sigma=\langle X, g\rangle$ is invisible if g. Handshake $\neq\{\}$ or g. Exclusive $\neq\{\}$. Any state that is not invisible is visible. The failure state $\Omega$ is always visible. Further $\Sigma=\langle X, g\rangle$ is dinvisible if $g$.dstep $=1$.

It is easy to show that every dinvisible state is also invisible. According to Page 98 of [Hol95], a dstep sequence is completely deterministic and any nondeterminism encountered may be resolved in an predetermined, but undefined way. The following definition covers this.

DEFINITION 5.6. $\rightarrow_{4}$ is a largest subset of $\rightarrow_{3}$ such that for every $\Sigma_{d}$, a dinvisible state the following property holds:

$$
\left(\Sigma_{d} \rightarrow_{4} \Sigma_{d}^{\prime} \wedge \Sigma_{d} \rightarrow_{4} \Sigma_{d}^{\prime \prime}\right) \Longrightarrow \Sigma_{d}^{\prime}=\Sigma_{d}^{\prime \prime}
$$


Clearly $\rightarrow_{4}$ is not unique; this corresponds to the possibility of resolving the nondeterminism in more than one way, The only requirement we have on this relation is that it is the largest subset of $\rightarrow_{3}$ satisfying the above mentioned property.

We are now finally in a position to define the relation $\rightarrow$ which exactly defines the execution steps of a PROMELA program.

DEFINITION 5.7. $\rightarrow$ is is the smallest binary relation over visible states defined as follows. If $\Sigma$ and $\Sigma^{\prime}$ are visible global states then

1. $\Sigma \rightarrow_{4} \Sigma^{\prime} \Longrightarrow \Sigma \rightarrow \Sigma^{\prime}$.

2. If $\Sigma_{1}, \cdots, \Sigma_{k}$ is a finite sequence of invisible states for some $k>0$ such that $\Sigma \rightarrow \Sigma_{1}$ and $\Sigma_{k} \rightarrow \Sigma^{\prime}$ and $\Sigma_{i} \rightarrow \Sigma_{i+1}$ for $1 \leq i \leq k$, then $\Sigma \rightarrow \Sigma^{\prime}$.

3. $\Sigma \rightarrow \Sigma$ if there exists an infinite sequence of invisible states $\left\{\Sigma_{i}\right\}$ and $\Sigma \rightarrow \Sigma_{0}$ and $\Sigma_{i} \rightarrow \Sigma_{i+1}$ for all $i \in$ Naturals.

\section{Example}

In this section, we illustrate by means of an example how a process declaration in textual form is represented as a symbolic labeled transition system.

Consider the following process type which is some arbitrary collection of PROMELA statements.

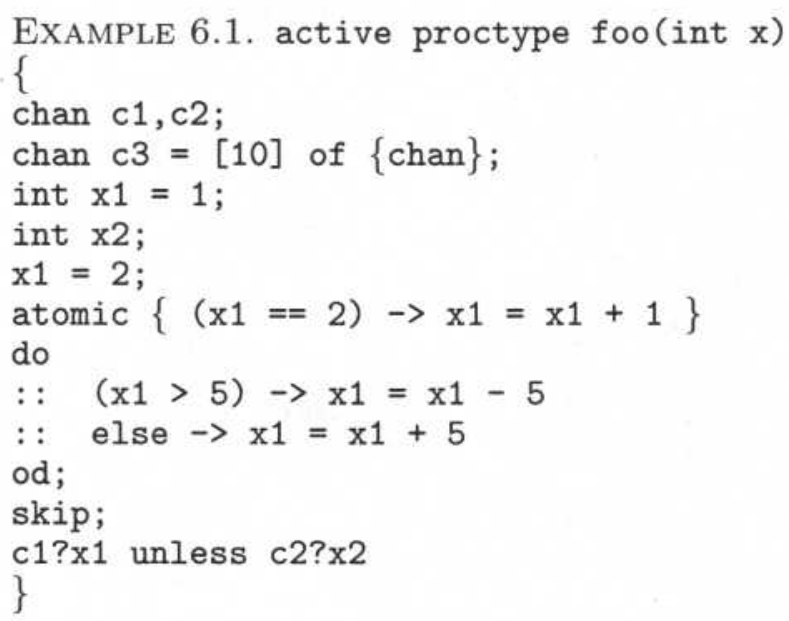

The symbolic labeled transition system corresponding to this program is $\left\langle\right.$ foo, Structure, $q_{0}$, locals, ChansOwned, $\left.1, x\right\rangle$

where Structure is given in Figure 1. locals is the partial function which takes values $0,0,1$ at $x, x 2, x 1$ respectively and undefined at all other arguments. ChansOwned is the finite sequence $\langle c 1, \Omega, \Omega\rangle,\langle c 2, \Omega, \Omega\rangle,\langle c 3,10$, chan $\rangle$.

The priority of the transition from state $q_{8}$ to $q_{11}$ is higher than the one from $q_{8}$ to $q_{9}$. The else statement is translated into the negation of the conjunction of the other guards of the same if or do statement. It is an error to have send or receive statements as alternatives to an else in a if or do statement. Statements that transfer control out of an atomic into a non-atomic region are executed in normal mode. 


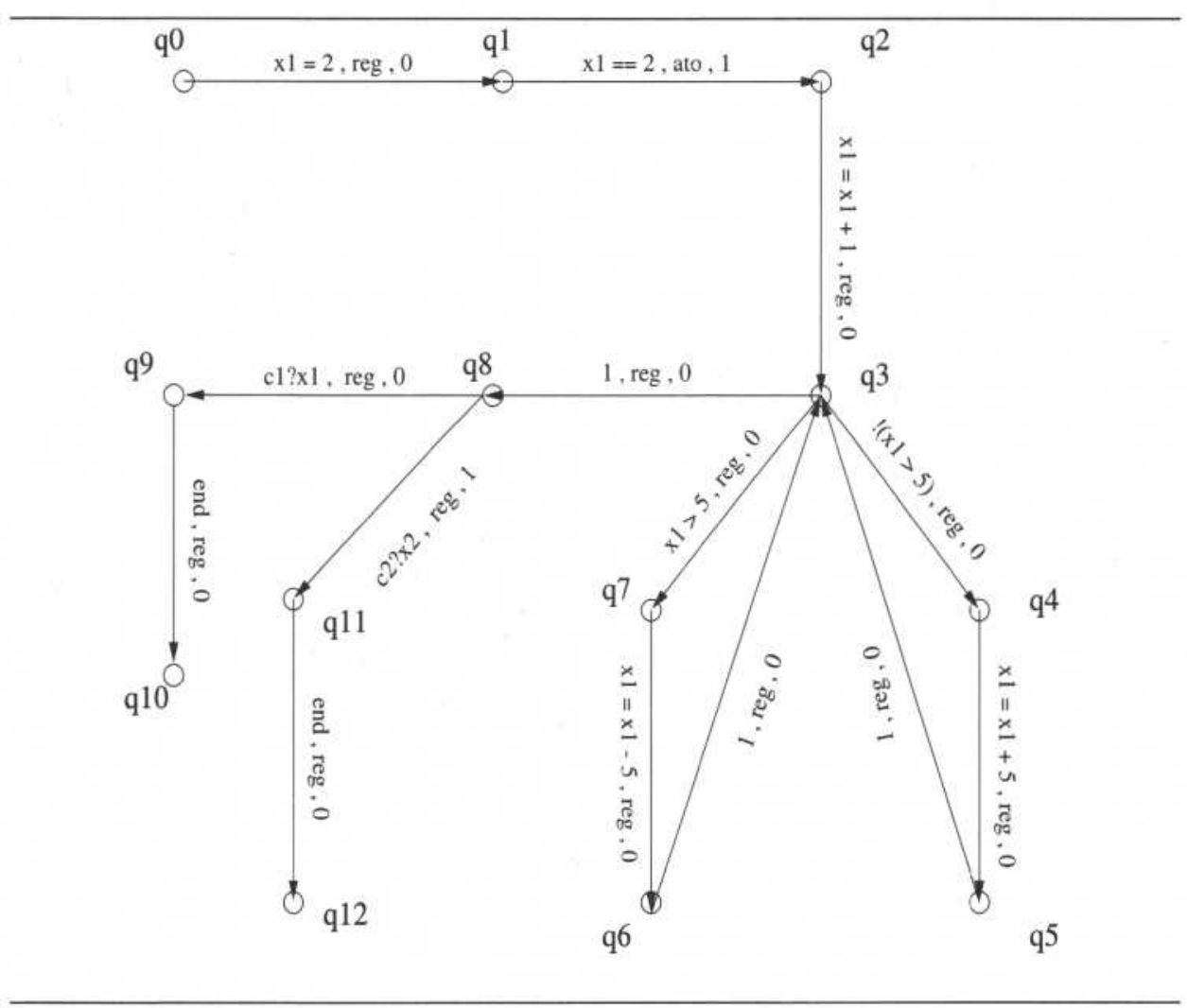

Figure 1. Sample Symbolic Labeled Transition System

\section{Summary}

This report gives an outline for an operational-semantics definition of the verification language Promela. We showed how a given Promela program, represented as sequence of symbolic labeled transition systems, can be translated into a Kripke structure. This was done by defining primitive statements as conditional state transformers. Not defined here is the conversion algorithm, part of SPIN, that translates PROMELA proctype definitions into symbolic labeled transition systems.

Features of the language that contribute to the complexity of the semantics include mobility (the ability to pass channel names over channels), and atomic and deterministic sequences. The first feature, for instance, necessitates the use of channel manipulation functions (Subsection 2.6.5) in the semantics of run and end statements.

For this outline we have made several simplifying assumptions about Promela syntax. For instance, we have not considered

- Correctness properties.

- Array variables.

- Data structures.

- Channels with more than one message field.

- Run statements with more than one argument. 
The semantics of the run statement, furthermore, is somewhat different from that presented in Page 95 of [Hol91]. In the semantics given here, the run statement cannot be used in composite arithmetic expressions. The semantics definitions can be modified fairly straightforwardly to overcome these restrictions.

\section{References}

[Hol91] G.J. Holzmann, Design and Validation of Computer Protocols, Prentice Hall, 1991.

[Hol94] G.J. Holzmann, What's New in SPIN Version 2, Online document, SPIN distribution, 1994.

[Hol95] G.J. Holzmann, The Verification of Concurrent Systems, Course Notes, To appear.

Networking Hardware Division, IBM Corporation, RTP, NC, USA

E-mail address: natarajraleigh.ibm.com

Bell Laboratories, Murray Hill, NJ, 07974 USA

E-mail address: gerardresearch.bell-labs.com 